\title{
Transdermal Scopolamine: Effects upon Psychological Performance and Visual Functioning at Sea
}

\author{
A. C. PARROTT $\dagger$ BSc, PhD \\ Senior Lecturer, Department of Psychology, North East London Polytechnic, London E15 4LZ, UK
}

\begin{abstract}
The effects of transdermal scopolamine upon psychological task performance, subjective feeling state, and visual functioning, were assessed with 28 naval volunteers at sea. The battery of assessment measures was given before drug administration, and following 24-hour periods on transdermal scopolamine and transdermal placebo. Code substitution task performance was not affected, but letter cancellation errors were significantly increased, indicating an impairment in sustained attention with transdermal scopolamine. Subjective reports of dry mouth and drowsiness were significantly more frequent under scopolamine. Visual changes were also noted, with visual near point significantly increased for the overall group, and one hypermetropic subject developing a marked visual acuity decrement and severe blurred vision, following transdermal scopolamine.
\end{abstract}

KEY wORDS - Scopolamine, acetylcholine, transdermal drug, psychological performance, attention, arousal, vision, visual near point.

\section{INTRODUCTION}

Scopolamine hydrobromide (hyoscine) has been frequently demonstrated to be the most effective single drug for the prophylaxis and treatment of motion sickness (Brand and Perry, 1966; Reason and Brand, 1975; Wood, 1979). One disadvantage with scopolamine as a motion sickness prophylaxis is its short duration of action following oral administration (5-6 hours; Innes and Nickerson, 1975; Wood, 1979). Repeated intermittent dosing is therefore necessary for sustained motion sickness protection, but this regime leads to pharmacokinetic variability, with changed levels of motion sickness protection over time. The recently developed transdermal scopolamine system was designed to deliver a steady low dose of scopolamine over an extended time period (Chandrasekaran, 1983; Clissold and Heel, 1986; Graybiel et al., 1976); when the patch is applied to the skin the drug slowly permeates from a central drug reservoir, through a rate-limiting membrane into the stratum corneum, and then enters the systemic circulation (Clissold and Heel, 1986; Shaw, 1983).

The effectiveness of transdermal scopolamine in reducing motion sickness has been demonstrated

†Author to whom correspondence should be addressed. under rough sea conditions (Price et al., 1981; Noy et al., 1984; Van Marian et al., 1985), and with laboratory-based nauseogenic motion devices (Dahl et al., 1984; Graybiel et al., 1976; Hordinsky et al., 1982, Homick et al., 1983). Neurochemical models of motion illness place primary emphasis upon muscarinic acetlycholine pathways, and the effectiveness of scopolamine in counteracting motion sickness reflects its strong anticholinergic properties (Innes and Nickerson, 1975; Kohl and Homick, 1983). Acetlycholine is, however, also involved in the neurotransmission of various psychological functions: the storage of new information into memory, sustained attention, vigilance, and arousal (Deutsch, 1971; Warburton and Wesnes, 1984). Oral and parenteral scopolamine have been demonstrated to impair each of these functions (Colquhoun, 1962; Lukomskya and Nikolskay, 1971; Poulton and Edwards, 1974; Ghoneim and Mewaldt, 1975; Drachman and Sahakian, 1979; Jones et al., 1979; Wesnes and Warburton, 1983, 1984; Parrott, 1986a). Although the effects of transdermal scopolamine upon psychological functions have been investigated, there is little agreement as to whether they are affected. Gordon et al. (1986) administered a mixed battery of psychological tasks and found no significant performance 
decrements. Hordinsky et al. (1982) reported an impairment with psychomotor tapping, while several other psychomotor and information processing tests were not affected. Parrott (1986a, 1987) found unimpaired psychomotor and information processing performance, but sustained attention, memory for new information, and feelings of alertness were significantly affected. Parrott and Jones (1985) also reported a significant sustained attention decrement. A central aim of the present study was therefore to further investigate psychological performance following transdermal scopolamine.

Acetylcholine is also involved in several autonomic nervous system functions, including control of the ciliary muscle holding the lens of the eye. Reduced cholinergic input following oral or parenteral scopolamine can lead to reduced visual accommodation and blurred vision (Innes and Nickerson, 1975), while visual problems have also been noted following transdermal scopolamine (Graybiel et al., 1976; Homick et al., 1983; Johnson et al., 1984; Parrott and Jones, 1985; Parrott, 1986b). Visual near point, visual acuity, and self reports of blurred vision, were therefore each investigated in the present trial.

\section{MATERIALS AND METHODS}

Subjects comprised 28 fully informed male volunteers from the crew of HMS Alacrity, in the age range $18-40$ years. All crew members who had used sea sickness medications in the past were screened as potential volunteers. Subjects were informed they could withdraw from the trial at any time, and that all data would be 'medical in confidence'. The trial was conducted in accordance with the Declaration of Helsinki. Alcohol was prohibited during the trial, and smoking was not allowed during testing; otherwise subjects went about their normal duties.

Drug conditions comprised standard transdermal scopolamine and transdermal placebo patches, identical in appearance. They were applied to the hairless skin behind the ear, following standard application procedures (Clissold and Heel, 1986). Patches were applied by the Medical Officer or Medical Assistant. The Medical Officer held sealed drug codes for possible emergencies; they did not need to be broken. Drug administration and testing were double-blind.

The psychological performance tests comprised code substitution and letter cancellation (Parrott, 1986a, 1987). Code substitution represents an information processing task; a series of letters was consecutively coded into numbers using a nine-item letter-number code. The average time per coding and percentage commission error were calculated. Letter cancellation represents a task requiring a high level of sustained attention for optimal target deletion; rows of randomly ordered letters were scanned, and each target letter identified (e.g. ' $T$ ') was deleted. The average time per letter scan, and percentage omission error were calculated. Response sheets and target letters or letter-number codes were changed at each test session. The duration of each test was 4 minutes. Choice reaction time was originally included as a performance test, but the apparatus broke down in the salt air and the test was abandoned.

The two visual assessments comprised visual near point and visual acuity. Visual near point was measured using the RAF near point rule (Price, 1978), the cursor being moved along the rule until it was reported to be just in focus. Each eye was tested separately, with the other eye screened behind a piece of grey card. The average of four readings from both eyes was taken (Parrott, 1986b). In the visual acuity test a Snellen chart was read at a constant distance, starting with the top row of largest letters, descending row by row through the letters steadily decreasing in size. An objective scoring system was devised which was based upon the last row totally correct (1=top row, 2=second row, 5=fifth row, $6=$ lowest row), and the percentage of correct letters in the next row containing error(s) $(5 \cdot 5=50$ per cent correct in the sixth row; $5 \cdot 8=80$ per cent correct in the sixth row). Each eye was tested separately and the average score calculated. Different Snellen charts were used at each test session. The subjective questionnaire covered various feelings over the past 16 hours (8-24 hours after patch application): vomiting, nausea, headache, blurred vision, dry mouth, drowsiness, skin irritation near the patch, and any other comments. Feelings at the moment were also covered: stomach awareness, ship motion judged unpleasant; difficulty in performing tasks, drowsiness, and social withdrawal (Table 2).

Subjects were given practice on all assessment measures before trial commencement; they also completed the full battery of assessment measures under normal testing conditions at the beginning of the trial. (Note: these were all treated as practice data, and not used in the analysis.) The first transdermal patch was then applied. On returning 24 
hours later the battery of assessment measures was undertaken. The old transdermal patch was then removed and a new patch applied. The subject returned 24 hours later, and the battery of assessment measures was again repeated. The time of testing was therefore held constant for each subject, but different subjects were tested at various times depending upon their work schedules.

The trial comprised a two-way crossover design. With two drug conditions (placebo, scopolamine), and two test sessions (first, second); half the subjects were tested in the order placebo-scopolamine, and half in the order scopolamine-placebo. The design was initially balanced, but one subject experienced severe blurred vision with transdermal scopolamine, and his data were withheld from all group analyses. This left an unbalanced design which was analysed by non-orthogonal analysis of variance for two-period crossover, with drug effects adjusted for treatment order, and order effects adjusted for treatment effect (Hills and Armitage, 1979). The third ANOVA factor was the group or interaction effect (i.e. between groups tested in the order scopolamine-placebo, and placebo-scopolamine; this is also described as the drug $\times$ order interaction (Hills and Armitage, 1979)). If a significant interaction factor is present in a two-way crossover trial, then the analysis can become complicated, with residual carryover effects, asymmetric transfer of learning, or unbalanced ceiling effects, possibly confounding drug or order effects (Hills and Armitage, 1979). There were, however, no significant interaction effects in the present ANOVAs. The subjective questionnaire data were analysed by the Gart test, a test appropriate for nominal data in a crossover design (Pethybridge, 1981).

\section{RESULTS}

The performance test findings are summarized in Table 1; they comprise the uncorrected group means from each drug condition. Letter cancellation omission errors were significantly more frequent under transdermal scopolamine, while letter cancellation times were significantly reduced (Table 1). Code substitution times and error rates were not significantly affected. Significant order effects were present with response time for both letter cancellation $(p<0.05)$ and code substitution $(p<0.001)$; order effects were not present with either letter cancellation error or code substitution error. There were no significant interaction effects. One subject experienced severe visual problems following
Table 1. Psychological performance assessment following transdermal scopolamine or transdermal placebo: group means and standard errors

\begin{tabular}{|c|c|c|c|c|}
\hline \multirow{2}{*}{$\begin{array}{l}\text { Performance measure } \\
\begin{array}{l}\text { Letter cancellation } \\
\text { time (seconds/letter) }\end{array}\end{array}$} & \multicolumn{2}{|c|}{$\begin{array}{l}\text { Transdermal } \\
\text { placebo }\end{array}$} & \multicolumn{2}{|c|}{$\begin{array}{l}\text { Transdermal } \\
\text { scopolamine }\end{array}$} \\
\hline & $0 \cdot 126$ & \pm 0.004 & $0 \cdot 12$ & $\pm 0.004^{*}$ \\
\hline $\begin{array}{l}\text { Letter cancellation } \\
\text { error }(\%)\end{array}$ & $7 \cdot 5$ & $\pm 1 \cdot 2$ & $9 \cdot 8$ & $\pm 1 \cdot 4^{*}$ \\
\hline $\begin{array}{l}\text { Code subsitution } \\
\text { time (seconds/coding) }\end{array}$ & 1.66 & \pm 0.04 & 1.69 & \pm 0.05 \\
\hline $\begin{array}{l}\text { Code substitution } \\
\text { error }(\%)\end{array}$ & 0.5 & $\pm 0 \cdot 1$ & $0 \cdot 8$ & \pm 0.3 \\
\hline $\begin{array}{l}\text { Visual near point } \\
\text { (centimetres) }\end{array}$ & $12 \cdot 0$ & \pm 0.5 & $12 \cdot 6$ & $\pm 0 \cdot 5^{*}$ \\
\hline $\begin{array}{l}\text { Visual acuity } \\
\text { (Snellen chart: } \\
\text { arbitrary scale) }\end{array}$ & $5 \cdot 7$ & $\pm 0 \cdot 1$ & $5 \cdot 6$ & $\pm 0 \cdot 1$ \\
\hline
\end{tabular}

Two-tailed significance level (ANOVA) ${ }^{*} p<0.05$

transdermal scopolamine. He complained of severe blurred vision, and his visual acuity (Snellen chart) score was reduced from 4.3 under placebo to 0.5 under scopolamine; his visual near point value under scopolamine could not be obtained due to the focusing difficulty. This subject was particularly hypermetropic, with a visual near point value of $22 \mathrm{~cm}$ under placebo, noticeably longer than any other subject (range $7-17 \mathrm{~cm}$, mean $11 \mathrm{~cm}$ ). With the remaining group of subjects, visual acuity values were not affected, but visual near point values were significantly lengthened following transdermal scopolamine $(p<0.05$, Table 1). Subjective feeling state values are presented in Table 2 . Dry mouth, and feelings of drowsiness when awake, were significantly more frequent following transdermal scopolamine. None of the other subjective reports differed significantly between drug conditions (Table 2).

\section{DISCUSSION}

Vomiting and feelings of nausea were infrequent under both transdermal scopolamine and transdermal placebo, reflecting the absence of severe sea conditions during trial (Table 2). Although sea sickness occurred infrequently, the trial situation did reflect a common naval scenario, with prophylactic medications being taken during moderate sea 
Table 2. Subjective self reports following transdermal scopolamine or transdermal placebo

\begin{tabular}{lcc}
\hline & \multicolumn{2}{c}{ Percentage reporting effect } \\
\cline { 2 - 3 } & $\begin{array}{c}\text { Transdermal } \\
\text { placebo }\end{array}$ & $\begin{array}{c}\text { Transdermal } \\
\text { scopolamine }\end{array}$ \\
\hline Feelings over past 16 hours & & \\
Vomiting & 4 & 14 \\
Feeling sick/nausea & 14 & 21 \\
Headache & 21 & 11 \\
Blurred vision & 7 & $50^{* *}$ \\
Dry mouth & 11 & $36^{*}$ \\
Drowsiness (when awake) & 14 & 7 \\
Other (negative statements) & 4 & 21 \\
Feelings at the moment & & 14 \\
Stomach awareness & 18 & 21 \\
Difficulty performing tasks & 0 & $2 \cdot 4$ \\
Ship motion judged unpleasant & 18 & $2 \cdot 3$ \\
Five point self rating scale (1-5): group mean values & & \\
Alert (1)-Drowsy (5) & $2 \cdot 1$ & \\
Sociable (1)-Withdrawn (5) & $2 \cdot 0$ & \\
\hline Two-tailed significance levels (Gart test) & & \\
* $p<0 \cdot 05$ & & \\
$*$ ** $p<0 \cdot 01$ & &
\end{tabular}

conditions, in case changes in sea state or ship heading led to more nauseogenic conditions prevailing.

With the psychological tests, letter cancellation omission errors were increased, while response times were decreased. The significant increase in omission errors has been reported in each land and sea trial using this measure (Parrott, 1986a, 1987; Parrott and Jones, 1985), confirming that sustained attention is impaired with transdermal scopolamine, as it is with oral and parenteral scopolamine (Colquhoun, 1962, Lukomskya and Nikolskay, 1971; Parrott, 1986a; Poulton and Edwards, 1974; Wesnes and Warburton, 1983, 1984). These attention decrements have occurred during two laboratory trials where smoking was restricted for 2 hours (30 minutes before testing, and 80-90 minutes during actual testing) (Parrott, 1986a, 1987); and during two sea trials where smoking was restricted only during 10 minutes of testing (Parrott and Jones, 1985; present trial). Future studies should directly investigate the effects of smoking or nicotine tablets during testing, to assess the extent to which the cholinergic agonist actions of nicotine counteract the antagonist actions of transdermal scopolamine (Wesnes and Warburton, 1984). The conclusion that transdermal scopolamine impairs attention contrasts with Gordon et al. (1986), who reported unimpaired performance on a protracted visual scanning or vigilance measure. However the task they used had not been demonstrated to be sensitive to psychoactive drug (or other stressor) effects; also the long duration of each stimulus target (20 seconds) was not typical of vigilance measures, where stimulus duration is generally short (Mackworth, 1957). The decreased letter cancellation time in the present study has not been previously reported, since non-significant response time increases have been previously noted (Parrott, 1986a, 1987; Parrott and Jones, 1985); although test materials, subject instructions, and test scoring, were constant across these studies. The data were therefore examined for a possible explanation, but none emerged. The possibility of a speed-error tradeoff was investigated, but the correlation between time change and error change was near to zero (Spearman $r=+0.07$, non-significant). Overall, therefore, the present response time decrease was atypical when compared with previous findings. The information processing task of code substitution was not impaired, confirming previous findings 
with coding tasks under transdermal scopolamine, both at sea (Parrott and Jones, 1985), and on land (Hordinsky et al., 1982; Gordon et al., 1986; Parrott, 1986a, 1987). Other performance tasks previously shown to be unaffected by transdermal scopolamine include cognitive tasks such as mental arithmetic, and psychomotor tasks such as discrete choice reaction time and target tracking (Hordinsky et al., 1982; Gordon et al., 1986; Parrott, 1986a, 1987). Psychological tasks significantly impaired by transdermal scopolamine include memory for new information (Parrott, 1986a; 1987), and psychomotor tapping (Hordinsky et al., 1982; although only at one of three post-drug test sessions). Although there can be problems in comparing findings from assessment measures which may vary in drug sensitivity, transdermal scopolamine does generally lead to impairments with tasks involving particular psychological functions; as with oral scopolamine, the tasks most sensitive to scopolamine involve sustained attention or memory for new information.

Feelings of daytime drowsiness were significantly increased under transdermal scopolamine (Table 2). Several land-based trials have previously reported significant drowsiness (Larsen and Peitersen, 1983; Gordon et al., 1986; Parrott, 1986a, 1987). However drowsiness has not generally been reported in sea trials (Noy et al., 1984; Parrott and Jones, 1985; Van Marian, 1985), while during land-based trials involving periods on laboratory motion devices, drowsiness sometimes been reported (Homick et al., 1983), and sometimes not (Dahl et al., 1984). There therefore seems to be an interaction between drug effects and motion, with the picture complicated by the difficulty of defining the degree of motion experienced. Also, changes in drowsiness under placebo during motion may be crucial in determining whether drug-placebo differences are statistically significant.

Visual changes were marked in one hypermetropic subject, with severe blurred vision, decreased visual acuity, and a focusing inability, following transdermal scopolamine. With the remaining subjects neither visual acuity nor self reports of blurred vision were significantly changed, but visual near point was significantly longer following scopolamine (Table 1). Visual problems have previously been reported with transdermal scopolamine. Graybiel et al. (1976) noted blurred vision in one of six subjects. Homick et al. (1983) reported that one of their subjects developed increasing visual problems following repeated patches. Johnson et al. (1984) described frequent blurred vision with cruise liner passengers. Parrott and Jones (1985) reported an inability to focus on written test materials with one subject following a single scopolamine patch, and in several subjects following successive patches. Parrott (1986b) demonstrated increased visual near point following a single patch although, as in the present study, blurred vision was not significantly affected following the first patch. Following successive patches, complaints of blurred vision increased and visual near points were further significantly lengthened, although only hypermetropic subjects were affected (Parrott, 1986b, figure 1). In conclusion, transdermal scopolamine does affect visual functioning; hypermetropic subjects are particularly at risk and may develop severe visual problems, while slight but consistent visual accommodation changes are also demonstrated in other subjects.

Subjective complaints of dry mouth were significantly increased with transdermal scopolamine (Table 2). This anticholinergic effect reflects reduced cholinergic input to the salivary glands, and has been noted in most studies investigating transdermal scopolamine (Price et al., 1981; Hordinsky et al., 1982; Homick et al., 1983; Dahl et al., 1984; Parrott and Jones, 1985; Van Marian et al, 1985; Gordon et al., 1986; Parrott, 1986a, 1987). There were no practical problems associated with patch use; none became accidentally dislodged, and skin irritation near the patch was not reported. Some of the subjects did, however, complain that they did not like being identified as sea sickness sufferers, suggesting that the patch could be applied on an area of skin covered by clothing. Unfortunately other sites are not recommended, since compared with the postauricuar area, percutaneous absorption is reduced by a factor of between 50 per cent (chest) and 95 per cent (thigh) in other skin sites (Chandrasekaran, 1983; Shaw, 1983). The problem of social acceptance of the transdermal system can, however, be illustrated from the outbreak of pirate patches during the trial; several crew members not participating as subjects, wore little paper circles taped behind their ears with the message: 'I don't get sea sick'!

In overall conclusion, transdermal scopolamine produces deleterious effects upon both central and autonomic nervous system functions. Tasks requiring a high level of sustained attention, or memory for new information, are generally impaired, while feelings of alertness may also be reduced. Visual 
changes also occur, which although mild with the majority of subjects, can be severe with hypermetropic individuals.

\section{ACKNOWLEDGEMENTS}

The author gratefully acknowledges the assistance provided by Surgeon Lieutenant-Commander T. J. R. Francis RN, former Medical Officer, HMS Alacrity; Dr R. J. Pethybridge, Chief Statistician, Institute of Naval Medicine; and $\mathrm{Mr} \mathrm{T}$. Beames, Experimental Assistant, Institute of Naval Medicine. Also Ciba-Geigy for the supply of transdermal patches.

\section{REFERENCES}

Brand, J. J. and Perry, W. L. M. (1966). Drugs used in motion sickness. Pharmacological Reviews, 18, 895-924.

Chandrasekaran, S. K. (1983). Controlled release of scopolamine for the prophylaxis of motion sickness. Drug Development and Industrial Pharmacy, 9, 627-646.

Clissold, S. P. and Heel, R. C. (1986). Transdermal scopolamine: a preliminary review of its pharmacodynamic properties and therapeutic efficacy. Drugs, 29, 189-207.

Colquhoun, W. P. (1962). Effects of hyoscine and meclozine on vigilance and short term memory. British Journal of Industrial Medicine, 19, 287-296.

Dahl, E., Offer-Ohlsen, D., Lillevold, P. E. and Sandvik, L. (1984). Transdermal scopolamine, oral meclozine and placebo in motion sickness. Clinical Pharmacology and Therapeutics, 36, 116-120.

Deutsch, J. A. (1972). The cholinergic synapse and the site of memory. Science, 174, 788-794.

Drachman, D. A. and Sahakian, B. J. (1979). Effects of cholinergic agents on human learning and memory. In: Nutrition and the Brain, vol. 5, Barbeau, A. (Ed.), Raven Press, New York, pp. 351-366.

Ghoneim, M. M. and Mewaldt, S. P. (1975). Effects of diazepam and scopolamine on storage, retrieval, and organisational processes in memory. Psychopharmacologia, 44, 257-262.

Gordon, C., Binah, O., Attias, J. and Rolnick, A. (1986). Transdermal scopolamine: human performance and side effects. Aviation Space and Environmental Medicine, 57, 236-240.

Graybiel, A., Knepton, J. and Shaw, J. (1976). Prevention of experimental motion sickness by scopolamine absorbed through the skin. Aviation Space and Environmental Medicine, 47, 1096-1100.

Hills, M. and Armitage, P. (1979). The two period crossover in clinical trials. British Journal of Clinical Pharmacology, 8, 7-20.
Homick, J. L., Lee-Kohl, R., Rescheke, M. F., Degionanni, J. and Cintron-Trevino, N. M. (1983). Transdermal scopolamine in prevention of motion sickness: evaluation of the time course efficacy. Aviation Space and Environmental Medicine, 54, 994-1000.

Hordinsky, J. R., Schwartz, E., Beier, J., Martin, J. and Aust, G. (1982). Relative efficacy of the proposed space shuttle antimotion sickness medications. Acta Astronautica, 9, 375-383.

Innes, I. R., and Nickerson, G. (1975). Atropine, scopolamine, and related antimuscarinic drugs. In: The Pharmacological Basis of Therapeutics, Goodman, L. S., and Gilman, A. (Eds), Macmillan, New York, 5th edn, pp. 514-532.

Johnson, P., Hansen, D., Matarazzo, D., Petterson, L., Swisher, C. and Trappolini, A. (1984). Transderm scop patches for the prevention of motion sickness. New England Journal of Medicine, 311, 468. (See also the reply by $R$. C. Steer, same reference).

Jones, D. M., Jones, M. E. L., Lewis, M. J. and Spriggs, T. L. B. (1979). Drugs and human memory: effects of low doses of nitrazepam and hyoscine on retention. British Journal of Clinical Pharmacology, 7, 479-483.

Kohl, R. E. and Homick, J. L. (1983). Motion sickness: a modulatory role for the central cholinergic nervous system. Neurosciences Biobehavioural Review, 7, $73-85$.

Lukomskya, N. Y. and Nikolskay, M. I. (1971). Search for drugs against motion sickness. Sechenov Institute, Leningrad. (Translation: Defence and Civil Institute of Environmental Medicine, Ontario, Canada).

Mackworth, N. H. (1957). Vigilance. The advancement of Science, 53, 389-393.

Noy, S., Shapira, S., Zilbiger, A. and Ribak, J. (1984). Transdermal therapeutic system scopolamine, dimenhydrinate and placebo, a comparative study at sea. Aviation Space and Environmental Medicine, 55, 1051-1054.

Parrott, A. C. (1986a). The effects of transdermal scopolamine and four dose levels of oral scopolamine $(0 \cdot 15$, $0 \cdot 3,0 \cdot 6$, and $1 \cdot 2 \mathrm{mg}$ ) upon psychological performance. Psychopharmacology, 89, 347-354.

Parrott, A. C. (1986b) Transdermal scopolamine: effects of single and repeated patches upon aspects of vision. Human Psychopharmacology, 1, 109-115.

Parrott, A. C. (1987). Transdermal scopolamine: effects of single and repeated patches upon psychological task performance. Neuropsychobiology, 17, 53-59.

Parrott, A. C. and Jones, R. (1985). Effects of transdermal scopolamine upon psychological test performance at sea. European Journal of Clinical Pharmacology, 28, 419-423.

Pethybridge, R. J. (1981). Some statistical tests for $2 \times 2$ tables. Journal of the Royal Naval Medical Service, 67, 37-42.

Poulton, E. C. and Edwards, R. S. (1974). Interactions range effects and comparisons between tasks in experiments measuring performance with pairs of 
stresses: mild heat and $1 \mathrm{mg}$ hyoscine hydrobromide. Aerospace Medicine, 45, 735-741.

Price, N. M., Schmitt, L. G., McGuire, J., Shaw, J. E. and Trobough, G. (1981). Transdermal delivery of scopolamine for the prevention of motion-induced nausea at sea. Clinical Pharmacology and Therapeutics, 29, 414-420.

Price, T. J. G. (1978). Ophthalmological conditions and eye examination. In: Aviation Medicine: vol. 2, Whiteside, T. C. D. (Ed.), Tri-med books, London, pp. 264--302.

Reason, J. T. and Brand, J. J. (1975). Motion Sickness. Academic Press, London.

Shaw, J. E. (1983). Development of transdermal therapeutic systems. Drug Development and Industrial Pharmacy, 9, 579-603.

Van Marian, W. F., Bongaerts, M. C. M., Christiaanse, J. C., Hofkamp, H. G. and Van Ouwerkerk, W. (1985).
Influence of transdermal scopolamine on motion sickness during 7 days' exposure to heavy seas. Clinical Pharmacology and Therapeutics, 38, 301-305.

Warburton, D. M. and Wesnes, K. (1984). Drugs as research tools in psychology: cholinergic drugs and information processing. Neuropsychobiology, 11, 121-132.

Wesnes, K. and Warburton, D. M. (1983). Effects of scopolamine on stimulus sensitivity and response bias in a visual vigilance task. Neuropsychobiology, 9, 154-157.

Wesnes, K., and Warburton, D. M. (1984). Effects of scopolamine and nicotine on human information processing performance. Psychopharmacology, 82, $147-150$.

Wood, C. D. (1979). Anti-motion sickness and antiemetic drugs. Drugs, 17, 471-479. 